\title{
Two effects of context on the presence/absence of connecting segments
}

\author{
DONALD L. KING and JONEIS THOMAS \\ Howard University, Washington, DC
}

\begin{abstract}
Stimulus A consisted of two proximal brackets with colinear lines separated by small gaps. Stimulus B was a square-like rectangle produced by inserting connecting segments into the gaps. Stimulus A was frequently represented as B (i.e., as closed). Following Pomerantz and Pristach (1989) and Treisman and Paterson (1984), perhaps A produced a closure emergent feature that was salient but otherwise independent of other features. However, adding the same two vertical lines (context) to both $A$ and $B$ produced a contrasting division outcome, even though the putative closure emergent feature was an element of the A+context stimulus, and even though it matched a physical feature of the $B+$ context stimulus. Therefore, this emergent feature did not produce the closure. Two additional experiments indicated that the two context lines made the two connecting segments more visible-a context-produced increase in visibility occurred that is not comparable to other known evidence of perceptual improvement. This greater visibility also indicates that the division was not due to the two context lines inhibiting the perception of the two connecting segments, and the closure was not due to good continuation.
\end{abstract}

Effects of context are central to perception. Context can inhibit (decrease the visibility of) another feature. It can also increase the extent to which another feature is perceived as being similar to the context (assimilation) or as being dissimilar to the context (contrast). Context also creates emergent features. For example, placing a dot near an already-present dot creates the emergent feature of the distance between these dots. Context also produces the apprehension of one or more, but a still-limited number of, perceived units (phenomenal wholes, experienced groups).

Context may directly affect the perception of another feature. For example, the ability of one contour to perceptually displace a proximal second contour away from it (and hence produce contrast) may be due to lateral inhibition from the first contour (Ganz, 1966). However, because context creates emergent features, effects of context may also be due to these features and/or associated factors. For instance, adding context may create a salient emergent feature, and this feature may strongly influence performance so that the influence of the original features are minimal (Pomerantz \& Pristach, 1989). Similarly, because context also contributes to the apprehension of perceived units, effects of context may be due to factors associated with these units. Perhaps, then, the same factor produces both the apprehension of a perceived unit

The authors would like to thank Howard Egeth and Steven Yantis for helpful discussions and methodological suggestions. This research was supported by the Howard University Faculty Research Support Grant Program and by National Institute of Mental Health ADAMHA-MARC Grant T-34-MH16580. Comelius Lewis and Elizabeth Phillips provided assistance with data collection and analysis. Please send correspondence concerning this article to D. L. King, Department of Psychology, Howard University, 525 Bryant St., N.W., Washington, DC 20059. and the "perceptual glue" that has been considered to connect the parts belonging to it.

The present paper relates to Pomerantz and Pristach's (1989) theory of context. However, due to an unexpected result, the present paper also involves the possibility that context can increase the visibility of a feature.

Pomerantz and Pristach (1989) maintain that context interferes with the perception of other features only because it creates salient emergent features, that is, emergent features that more quickly affect perceptual performance than do other features. Otherwise, emergent features function independently of other features; emergent features do not inhibit or otherwise directly affect the perceptual influence of other features. This is a salience-independence theory of context.

The stimuli in Figure 1 were formed by combining a left vertical angle (A1) or a right vertical angle (A2) with either of two diagonal lines (B1 and B2). Pomerant, Sager, and Stoever (1977) found better discrimination between one of these triangles and one of these arrows than between their component diagonal lines, even though the identical right angle was the second component of both the triangle and the arrow. In one classification task of Pomerantz and Pristach (1989), the two objects with the Al feature required Response $1(R 1)$, and the two objects with the A2 feature required Response 2 (R2). A second classification task assigned R1 to the two $B 1$ objects, and $R 2$ to the two B2 objects. Performance on both of these tasks was poor. In contrast, performance on a third classification task, in which $\mathrm{AlB} 2$ and $\mathrm{A} 2 \mathrm{~B} 1$ were assigned to $\mathrm{R} 1$, and $A 1 B 1$ and $A 2 B 2$ were assigned to $R 2$, was good.

Pomerantz et al. (1977) maintained that joining a diagonal line and a right angle to make a triangle or arrow produced emergent features. In addition, these features 


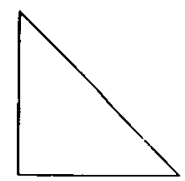

A1B1

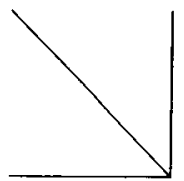

А2B1

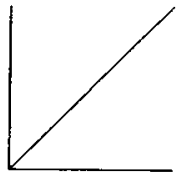

A1B2

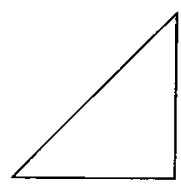

A2B2
Figure 1. Representations of four stimuli employed by Pomerantz and Pristach (1989).

were novel and highly discriminable (and hence more salient) than the features of the diagonal lines, explaining why the performance on the triangle-arrow discrimination was better than that on the diagonal-diagonal discrimination. Pomerantz and Pristach (1989) also posited that this joining produced salient emergent features. In addition, the poor performances on the first two (i.e., Al vs. $\mathrm{A} 2$ and $\mathrm{B} 1$ vs. B2) classifications were due to the uselessness of the emergent-feature difference that distinguishes a triangle from an arrow, due to one triangle and one arrow being assigned to $\mathrm{R} 1$ and the remaining triangle and arrow being assigned to R2. And the good performance on the third classification task occurred because the same emergent-feature difference was now useful, due to two objects of the same shape being assigned to the same response. Realize that Pomerantz and Pristach did not attribute the poor classification performance to interference with the perception of the features that required different responses (e.g., A1 and A2). Emergent features affected performance only because they were highly salient.

Pomerantz and Pristach (1989) also considered the possibility that each object (one triangle or one arrow) functions as a unit; perceptual glue may connect all or most of the features of each object. If so, it should be difficult to perceive an object's individual features, including those features requiring different responses (e.g., A1 and A2). Therefore, they replaced one of the triangles and one of the arrows in Figure 1 with ones that were sufficiently similar that the apparent strengths of the perceived units that the old and new objects produced were essentially identical. Nevertheless, this second experiment produced a new pattern of classification results. Therefore, Pomerantz and Pristach concluded that their classification results cannot be explained in terms of perceived units and perceptual glue.
Treisman and Paterson's (1984) individual objects approximated the triangles, arrows, right angles, and diagonal lines of Pomerantz and Pristach (1989) and Pomerantz et al. (1977). Each display consisted of one triangle, one arrow, or neither of these objects, along with multiple right angles and diagonal lines. Identifying the triangle produced fewer illusory conjunctions of a right angle and a diagonal line than did identifying the arrow. This result may have occurred because joining a right angle and a diagonal line to make a triangle produces the distinctive emergent feature of closure. This interpretation was backed with search and texture segregation results, and the finding that regular (and hence closed) circles increased the false identifications of triangles. Treisman and Paterson concluded that a triangle affects performance because it produces the perceptually primitive emergent feature of closure. Moreover, this emergent feature is influential, because of its preattentive availability (similar to high saliency), rather than because it affects (e.g., inhibits) the perception of other features. This parallels what has been labeled here as the salience-independence theory.

So, the results of classification, illusory conjunction, and other types of tasks support the salience-independence theory. Moreover, this theory is consistent with the hypothesis that a limited number of different types of perceptual primitives (elementary features) are registered preattentively and thereby affect perceptual performance (Julesz, 1984; Treisman \& Gelade, 1980). Furthermore, salienceindependence theory has received recent attention (Banks \& Krajicek, 1991), partly because it provides an alternative to positing that the features of an object are difficult to perceive because they are connected by perceptual glue.

Therefore, the purpose of Experiments 1 and 2 was to test the salience-independence theory. A "division" outcome questioned this theory. The purpose of Experiment 3 was to test a context-produced inhibition explanation of this outcome. Surprisingly, evidence of a context-produced increase in visibility was obtained instead, as confirmed by Experiment 4 .

\section{EXPERIMENT 1}

A salient emergent feature should remain influential despite the addition of a small amount of contour. Similarly, perceptual primitives are assumed to be registered without mention of context-produced interference (Julesz, 1984; Treisman, 1986). Therefore, the general strategy was to find out if adding contour (context) would affect a perceptual outcome that could be attributed to a salient emergent feature. In accord with Treisman and Paterson (1984), the putative emergent feature of closure was investigated.

The top row of Figure 2 shows photocopies of the two brackets and the square-like rectangle (Pair 1) and the two upright rectangles and the internal-lines rectangle (Pair 2) that were employed. The two brackets are readily apprehended as the square-like rectangle, so the two brackets could well be frequently falsely identified as this rectangle; closure (an illusory connection) could be expected. The 


\section{PAIR 1}

EXPT. 1

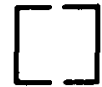

$3.38(3.47)$

EXPT. 2

EXPT. 3

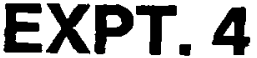

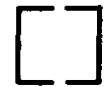

$530(0.80)$<smiles>C=C</smiles>

$1.05(1.20)$<smiles>C=C</smiles>

10.08

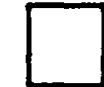

$1.00(1.13)$

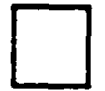

$414(0.00)$<smiles>[CH]=C</smiles>

$1.75(1.75)$<smiles>C=C</smiles>

8.17

PAIR 2

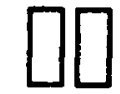

$1.92(2.29)$

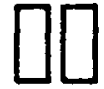

$454(0.20)$

BLANK

$0.25(0.45)$

BLANK

1.67

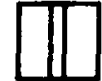

3.79 (3.92)

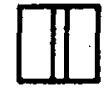

635 (1.50)

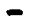

$5.25(5.30)$

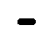

23.67

Figure 2. From left to right, the stimuli for Experiments 1 and 2 are the two brackets, the square-like rectangle, the two upright rectangles, and the internal-lines rectangle (although Experiment 2 employed additional stimuli). The stimuli for Experiments 3 and 4 are the two vertical lines, the very narrow rectangle, a blank (empty card), and the two 2-mm segments. For Experiments 1 and 3, the mean number of false identifications of each stimulus as the other member of its pair and the mean number of total false identifications for each stimulus (in parentheses) are indicated. For Experiment 4, only the former type of false identification is indicated (because each stimulus could be falsely identified as only one other stimulus). For Experiment 2, the mean reaction time (in milliseconds) and the mean number of total false identifications (in parentheses) for each stimulus are indicated.

salience-independence theory would attribute this result to the two brackets' producing a closure emergent feature that was more salient than the features that indicate two spatially separated objects.

Pair 2 was formed by adding two identical vertical lines to each Pair 1 object. Thus, the two brackets and the squarelike rectangle are A and B, respectively, and the two upright rectangles and the internal-lines rectangle are $\mathrm{A}+$ context and $\mathrm{B}+$ context, respectively. The salienceindependence theory assumes that the two brackets produce a salient closure emergent feature and that this feature should not be inhibited by adding a small amount of context. Therefore, the salience-independence theory predicts that if the two brackets result in closure, the two upright rectangles should also result in closure.

Accordingly, Experiment 1 determined whether both Pair 1 and Pair 2 would result in closure. Each object was presented very briefly, all four objects were choices, and the task was to identify the object that occurred.

\section{Method}

Stimuli. Figure 2 shows that (1) single identical vertical lines were added to each bracket to produce each upright rectangle, and (2) identical short segments were inserted in the identical gaps between the two brackets and the two upright rectangles to produce the squarelike rectangle and the internal-lines rectangle, respectively.

Each stimulus was drawn in black ink on a white card. Each vertical line was $11 \mathrm{~mm}\left(0.75^{\circ}\right)$ long. Each horizontal line of a bracket or upright rectangle was $5 \mathrm{~mm}\left(0.34^{\circ}\right)$ long. The top and bottom gaps between the two brackets and the two upright rectangles were $2 \mathrm{~mm}\left(0.14^{\circ}\right)$ long, making the inserted segments also $2 \mathrm{~mm}$ long. Each stimulus was centered on the card.

The identical stimuli were also choices. They appeared on a $21.6 \mathrm{~cm}(8 \mathrm{1} / 2 \mathrm{in}.) \times 27.9 \mathrm{~cm}(11$ in. $)$ sheet of white paper. The two brackets and the square-like rectangle of Pair 1 were to the left and right, respectively, as were the two upright rectangles and the internal-lines rectangle of Pair 2, respectively. Either Pair 1 or Pair 2 was at the top of the choice sheet. For both sheets, the numbers $1,2,3$, and 4 appeared below the stimulus in the top left, top right, bottom left, and bottom right locations, respectively.

Procedure. A Gerbrands G1132 tachistoscope was used. A 1-sec visual warning stimulus, a $7.6 \mathrm{~cm}\left(5.18^{\circ}\right)$ high $\times 12.7 \mathrm{~cm}\left(8.66^{\circ}\right)$ wide gray square centered on a black background, was followed by $1 \mathrm{sec}$ of darkness and then by one of the experimental stimuli. The luminance of the white background was approximately $6.9 \mathrm{~cd} / \mathrm{m}^{2}$. The stimulus duration of each stimulus was decreased over trials according to one of two fixed schedules: (1) $20,15,10,5,3,2$, and $1 \mathrm{msec}$ for blocks of Trials $1-4,5-8,9-12,13-16,17-32$, 33-48, and 49-64, respectively, and (2) 10, 5, 3, 2, and $1 \mathrm{msec}$ for Trials $1-4,5-8,9-16,17-32$, and 33-48, respectively. For each of these blocks, each of the four stimuli occurred on one fourth 
of the trials in a random order. The purpose of using relatively long initial durations was to make the stimuli clearly identifiable, so that subjects would understand the task before it became more difficult.

At the termination of a stimulus, the subject indicated his/her choice by speaking its number. There was no feedback.

Subjects and Groups. There were 2 (Schedule 1 or Schedule 2) $\times 2$ (choice sheet: 1 or 2 ) $=4$ independent groups. The 6 subjects in each group were students in an introductory psychology class at Howard University. Participation contributed to satisfying a course requirement.

\section{Results}

The first four trials of the experiment were disregarded. In addition, if the number of correct responses on the 16trial blocks was not 9 or over, the blocks were disregarded. The rationale for this practice was that relatively frequent errors would be avoided, because they should be associated with more nearly random choice behavior, which would add to error variance. A total of seven such blocks were disregarded. In the related Experiment 3, a total of three such blocks were disregarded.

For each subject, the false identifications of each stimulus as the other member of its pair were summed across trials. For example, the false identifications of the two brackets as the square-like rectangle were summed across trials. The first row of Figure 2 indicates the mean number of these false identifications for each stimulus. The mean of the total number of false identifications of each stimulus is shown in parentheses.

Note that the mean number of false identifications of each stimulus as the other member of its pair was almost as great as the mean number of total false identifications of each stimulus. Therefore, the experiment essentially involved discriminating each stimulus from the other member of its pair. Consequently, the sums of the false identifications of each stimulus as the other member of its pair were the scores for an analysis of variance (ANOVA), which was a 2 (pairs: Pair 1 or 2$) \times 2$ (2-mm segments: present or absent) $\times 2$ (schedule: 1 or 2 ) $\times 2$ (choice sheet: 1 or 2 ) factorial. Only the last two factors were between subjects.

The pairs $\times 2-\mathrm{mm}$ segments interaction was significant $[F(1,20)=16.25, p<.001]$. For Pair 1 , the mean number of false identifications of the two brackets as the square-like rectangle was 3.38 , and the mean number of false identifications of the square-like rectangle as the two brackets was $1.00[F(1,20)=10.19, p<.01]$. But for Pair 2, the mean number of false identifications of the two upright rectangles as the internal-lines rectangle was 1.92 , and the mean number of false identifications of the internallines rectangle as the two upright rectangles was 3.79 $[F(1,20)=6.29, p<.05]$. Therefore, the subjects tended to incorrectly close the two brackets, but tended to incorrectly divide the internal-lines rectangle.

The only other significant result was an uninteresting pairs $\times$ schedule interaction $[F(1,20)=4.73, p<.05]$.

\section{Discussion}

The two brackets tended to be falsely identified as the square-like rectangle; closure occurred. However, even though the two upright rectangles contain the two brackets, and hence the salient emergent feature that the salienceindependence theory assumes underlies closure, the two upright rectangles did not lead to closure. Therefore, the salience-independence prediction was disconfirmed.

In fact, the internal-lines rectangle tended to be falsely identified as the two upright rectangles-a division outcome that is a reversal of the salience-independence prediction. Treisman and Schmidt (1982, pp. 110-111) consider the possibility that perceptual primitives reduce the influence of other perceptual primitives. But assuming that adding the two lines to the two brackets reduced the influence of the putative closure emergent feature still does not account for the reversal.

Moreover, the internal-lines rectangle contains the closed square-like rectangle as a physical element. Therefore, the internal-lines rectangle possesses the physical feature of closure. A physical feature should be at least as salient as a matching emergent feature. Consequently, the intemallines rectangle should not have been frequently falsely identified as divided. In conclusion, in opposition to the salience-independence theory, the closure outcome was probably not due to the two brackets' producing a putative closure emergent feature.

Moreover, the closure and division outcomes cannot be readily attributed to several well-known, and hence uninteresting, factors. Because the members of both pairs differed only in the presence/absence of the connecting 2-mm segments, (1) the subjects presumably compared the clarity of the 2-mm segments that both the two Pair 1 stimuli and the two Pair 2 stimuli produced, and (2) the subjects presumably attended equally strongly to the location of the gap and the 2 -mm segments. In addition, Pairs 1 and 2 were highly similar in other ways (below), which should have produced the expectation of comparable outcomes, rather than the obtained opposing closure and division outcomes. Therefore, these outcomes should not have been due to a difference in the features that were compared, a difference in attention to these features, or a difference in expectation.

The two Pair 1 stimuli are about as similar to each other in features as are the two Pair 2 stimuli: the two brackets almost match the two upright rectangles, the single lines that were added to each bracket to make each rectangle are identical, the two bracket/line relations are virtually identical, and the $2-\mathrm{mm}$ segments that were added to both the two brackets and the two upright rectangles are identical. Therefore, it is difficult to attribute the opposing closure and division outcomes to a difference in similarity in features.

Also, consider that a perceptual dimension from one object (or no gap) to two objects (or gap) underlay performance. If so, then placing a decision criterion close to the end of the dimension with two objects would make the two brackets frequently be falsely identified as the square-like rectangle, as obtained. But then the two upright rectangles would frequently be falsely identified as the internal-lines rectangle as well, which is the reverse of the division outcome. 


\section{EXPERIMENT 2}

Two very similar brackets were difficult to identify as separate objects (King, 1989, 1990a). They were also frequently drawn as one square-like object (King, 1989). Gillam (1975) also obtained a closure type of result with proximal colinear lines.

However, there is no additional support for the more critical division outcome. Therefore, the primary purpose of Experiment 2 was to determine if an essentially identical division outcome could be obtained with the same stimuli that were used in Experiment 1.

A speeded one-object versus two-object task was used. The stimuli remained present while the response was made, which limited memorial and cognitive interpretations of the results.

Following previous work with the one-object versus two-object task (King, 1989, 1990a), (1) the two-object stimuli were also far apart, and (2) the one-object stimuli included each individual object of the two-object stimuli. Nevertheless, these additional stimuli were not germane to the replicability of the division outcome.

\section{Method}

Stimuli. There were 2 (brackets or upright rectangles) $\times 2$ ("near" or "far") two-object stimuli. These stimuli were the same as those used in Experiment 1, except that the gap between the two objects of each far stimulus was $22 \mathrm{~mm}$. The connected one-object stimuli included the square-like rectangle and internal-lines rectangle of Experiment 1. They also included the large rectangle and the large internal-lines rectangle produced by connecting the two far brackets and the two far upright rectangles with $22-\mathrm{mm}$ top and bottom segments, respectively. The individual one-object stimuli were the individual objects of the two-object stimuli. There were 2 (bracket or rectangle) $\times 2$ (near or far) $\times 2$ (left or right) such stimuli.

Groups and Procedure. Ten new subjects were instructed to respond to the two-object stimuli but not to the one-object stimuli, and 10 additional subjects received the reverse instructions. One reason that this go/no-go task was used is that the two-object task may result in one type of encoding, and the one-object task may result in a second type of encoding (King, 1992). Therefore, with the more typical binary response procedure, each response may be affected by two encodings: (1) its own go/no-go encoding, and (2) the go/no-go encoding for the alternative response, due to responding by default, which could complicate analysis.

The four connected one-object stimuli and the four two-object stimuli occurred twice in each of two consecutive blocks of 16 trials. The eight individual one-object stimuli occurred once, and the four two-object stimuli occurred twice in each of two additional consecutive blocks of 16 trials. Half of both the two-object and oneobject groups received the block with the connected one-object stimuli first, and the other half received the block with the individual one-object stimuli first. Both types of blocks were preceded by a block of eight practice trials, with stimuli selected randomly from the same type of block. The second type of 16-trial block (and also the preceding practice trials) followed the first without delay. Otherwise, the method followed that used in Experiment 1.

\section{Results}

Only the responses to the stimuli of Experiment 1 were analyzed. The reaction times (RTs) and errors to each of these stimuli were summed across the two blocks in which they both occurred. The second row in Figure 2 indicates the resulting mean RTs (in milliseconds) and errors. Note that errors on the one-object stimuli were made by the subjects instructed to respond to the two-object stimuli, and vice versa, meaning that the RTs and errors to the same stimulus were made by different groups.

The ANOVA was a 2 (pairs: Pair 1 or 2 ) $\times 2[2-\mathrm{mm}$ segments: present (one-object stimulus) or absent (twoobject stimulus)] $\times 2$ (order of blocks: connected oneobject blocks first, individual one-object blocks first) factorial for both RTs and errors. Only the last two factors were between subjects.

For the RTs, a significant pairs $\times 2$-mm segments interaction occurred $[F(1,16)=128.17, p<.001]$. The square-like rectangle produced a mean RT of $414 \mathrm{msec}$, and the two brackets produced a mean RT of $530 \mathrm{msec}$ $[F=14.82, p<.01]$. In contrast, the internal-lines rectangle produced a mean RT of $635 \mathrm{msec}$, and the two upright rectangles produced a mean RT of $454 \mathrm{msec}$ $[F(1,16)=35.95, p<.001]$. Furthermore, the error ANOVA yielded a nearly equivalent pairs $\times 2$-mm segments interaction $[F(1,16)=25.94, p<.001]$.

\section{Discussion}

These results indicate that the internal-lines rectangle produced a poorer performance than did the two upright rectangles. The division outcome of Experiment 1 suggests that the internal-lines rectangle produced a representation of two upright rectangles early in processing. This representation should interfere with the present experiment's task of indicating that the internal-lines rectangle is a one-object stimulus, thereby accounting for the poor performance on this rectangle. Therefore, the present experiment corroborates the division outcome of Experiment 1 . These results also indicate that the square-like rectangle produced a better performance than did the two near brackets, which similarly corroborates the closure outcome of Experiment 1.

The present evidence against the salience-independence theory was obtained with stimuli that remained present until the response was made. In addition, there was no freedom of choice and there was no time for deliberation, also unlike in Experiment 1. Therefore, the present evidence should not have been affected by a memorial process, and if a bias were influential, it should have been perceptual.

\section{EXPERIMENT 3}

A dominant-subordinate type of interaction, in which a dominant object inhibits the perception of a subordinate object, occurs when two objects differ in intensity (Weisstein, 1972, pp. 234-235) and size (King, 1990b). The 11:2 ratio in size of the internal lines to connecting segments of the internal-lines rectangle hints that a similar dominant-subordinate interaction produced the division outcome. That is, these internal lines may have been dominant, and hence may have inhibited the perception of 
these connecting segments, thereby producing the division outcome.

To test this inhibition possibility, the stimuli in the third row of Figure 2 were employed. The right-hand member of Pair 1 , the very narrow rectangle, is identical to the two internal lines and the two 2-mm segments of the internal-lines rectangle. The left-hand member of Pair 1 consists of two vertical lines that are identical to the two internal lines of the internal-lines rectangle (except that these vertical lines are no longer internal). Pair 2 consists of the 2-mm segments alone and a blank (empty) card. The task was to identify the stimulus that occurred. The inhibition possibility maintains that the internal lines of the internal-lines rectangle inhibited (erased) the perception of the 2-mm segments. If so, then the very narrow rectangle's vertical lines should inhibit the perception of its $2-\mathrm{mm}$ segments. Therefore, it was predicted that the very narrow rectangle would be falsely identified as the vertical lines more frequently than the $2-\mathrm{mm}$ segments would be falsely identified as the blank. Surprisingly, the reverse result occurred.

\section{Method}

The method was identical to that for Experiment 1, except as follows. Only Schedule 1 of Experiment 1 was used to reduce the stimulus duration, because (1) for the first several trials, Schedule 1 resulted in fewer errors than did Schedule 2, which could improve subjects' understanding of the task, and (2) Schedule 1 contained more trials than did Schedule 2. Therefore, there were only two independent groups, each with 10 new subjects. Either Pair 1 or Pair 2 was at the top of the choice sheet. For both of these pairs, the stimulus without the $2-\mathrm{mm}$ segments was on the left.

\section{Results}

The third row in Figure 2 shows the mean number of false identifications of each stimulus as the other member of its pair, and the mean number of total false identifications of each stimulus (in parentheses). Note that the mean number of false identifications of each stimulus as the other member of its pair was almost as great as the mean number of total false identifications of each stimulus. Therefore, essentially, there were two tasks: to discriminate between the two Pair 1 stimuli, and to discriminate between the two Pair 2 stimuli. Consequently, the scores for the ANOVA were the sum of the false identifications of each stimulus as the other member of its pair, as in Experiment 1 . The ANOVA was a 2 (pairs: Pair 1 or 2 ) $\times 2$ (2-mm segments: present or absent) $\times 2$ (choice sheet: 1 or 2) factorial. Only the last factor was between subjects.

Most importantly, the mean number of false identifications for Pair $1[(1.05+1.75) / 2]$ was less than for Pair 2 $[(0.25+5.25) / 2][F(1,18)=16.71, p<.001]$. In addition, the pairs $\times 2$-mm segments interaction was significant $[F(1,18)=30.12, p<.001]$. This interaction occurred because the 2-mm segments were much more likely to be falsely identified as the blank than vice versa. Therefore, a main effect of $2-\mathrm{mm}$ segments also occurred $[F(1,18)=47.74, p<.001]$.

\section{Discussion}

The 2-mm segments were highly likely to be falsely identified as the blank, which is the reverse of the predicted result. Therefore, the possibility that the internal lines of the internal-lines rectangle inhibited the perception of the 2-mm segments was directly opposed.

The Pair 1 (very narrow rectangle and vertical lines) discrimination was easier than the Pair 2 (2-mm segments and blank) discrimination. The two members of each pair differed by the presence/absence of the identical $2-\mathrm{mm}$ segments. These segments occurred alone. Therefore, the relatively strong tendency for the subjects to falsely identify these segments as the blank could not have been due to context-produced interference. Consequently, the very narrow rectangle's vertical lines (context) should have improved the perception of its $2-\mathrm{mm}$ segments. So, evidence that context can increase the visibility of a feature was obtained.

\section{EXPERIMENT 4}

The purpose of Experiment 4 was to determine if stronger evidence of a context-produced increase in visibility could be obtained. In one stage (either first or second), the subjects discriminated between the Pair 1 stimuli-the very narrow rectangle and the vertical lines. In the other stage, they discriminated between the Pair 2 stimuli-the $2-\mathrm{mm}$ segments and the blank.

\section{Method}

The method was the same as that used for Experiment 3, except as follows.

Stimuli and Design. The stimuli were prepared with a computer drawing program. The new subjects discriminated between the Pair 1 stimuli in an initial stage, and the Pair 2 stimuli in a subsequent stage, or vice versa. The choice sheet for each stage contained the two stimuli of a pair; the left stimulus was labeled " 1 ," and the right stimulus was labeled " 2 ."

The two stimuli with the $2-\mathrm{mm}$ segments (i.e., the very narrow rectangle and the $2-\mathrm{mm}$ segments) were on the same side (left or right) of the choice sheet in both stages, and the two stimuli without these segments were on the other side. Three subjects received each combination of the two levels of the stage and choice sheet factors, making the total sample size 12 .

Procedure. For both stages, five successive blocks of 4 trials each were followed by five successive blocks of 16 trials each. Each member of the pair occurred equally often in each block. For the 4-trial blocks, the stimulus duration decreased from $5 \mathrm{msec}$ to $1 \mathrm{msec}$ in 1-msec steps over the five successive blocks.

With respect to the Stage 1 16-trial blocks, three or fewer errors on any of the first four 16-trial blocks led to a more difficult discrimination on the following block. This was accomplished by decreasing the duration of the stimulus by $1 \mathrm{msec}$, when the stimulus duration had been previously increased. This was accomplished by decreasing the luminance of the white background to, first, approximately $5.6 \mathrm{~cd} / \mathrm{m}^{2}$, then $2.8 \mathrm{~cd} / \mathrm{m}^{2}$, then $1.7 \mathrm{~cd} / \mathrm{m}^{2}$, and then $1.2 \mathrm{~cd} / \mathrm{m}^{2}$, when the stimulus duration was $1 \mathrm{msec}$ (and hence could not be reduced further). Also, four to six errors on any of the first four 16-trial blocks led to the same duration and intensity for the following block. In addition, seven or more errors on any of the first four 16-trial blocks led to an easier discrimination on the fol- 
lowing block. This was accomplished by increasing the duration of the stimulus by $1 \mathrm{msec}$ or, when the intensity had previously been reduced, by increasing the intensity to the next level.

After the last Stage 1 block, the subject received the choice sheet with the second pair of stimuli. The duration and intensity for each block of Stage 2 was equal to that of the corresponding block of Stage 1; stimulus duration and intensity were equal for the two stages.

\section{Results}

The scores for the ANOVA were the sum of the false identifications of each stimulus as the other member of its pair (as previously). The fourth row in Figure 2 indicates the resulting mean false identifications for each stimulus. The ANOVA was a 2 (pair: Pair 1 or 2$) \times 2(2-$ mm segments: present or absent) $\times 2$ (order: Pair 1 or 2 in Stage 1) $\times 2$ (choice sheet: 1 or 2 ) factorial. Only the order and choice sheet factors were between subjects.

Most importantly, Pair 1 produced fewer false identifications $[(10.08+8.17) / 2]$ than did Pair $2[(1.67+23.67) / 2]$ $[F(1,8)=5.67, p<.05]$. Due to 1 atypical subject, the significance level improved with a nonparametric test: For 11 out of 12 subjects, the Pair 1 mean was less than the Pair 2 mean $(p<.01$, two-tailed, sign test).

The pair $\times 2$-mm segments interaction was also significant $[F(1,8)=56.69, p<.001]$, due to the much greater tendency for the subjects to falsely identify the 2 -mm segments as the blank than vice versa. The same tendency was responsible for a main effect of 2-mm segments $[F(1,8)=57.68, p<.001]$.

\section{Discussion}

The vertical lines duplicate a large proportion of the perimeter of the very narrow rectangle, whereas the 2$\mathrm{mm}$ segments and the blank are categorically different. Therefore, the better discrimination on Pair 1 than on Pair 2 was not due to the two stimuli with less similar features producing the better performance. In addition, because total errors were compared, the distribution of these errors on either side of a decision criterion is not an issue. Furthermore, context could not have interfered with the perception of the 2-mm segments, because these segments occurred alone. Moreover, the only features that accompanied the 2-mm segments of the very narrow rectangle were the two vertical lines. Consequently, these vertical lines must have improved the perception of these 2$\mathrm{mm}$ segments. Also, the discrimination between the 2$\mathrm{mm}$ segments and the blank did not require the $2-\mathrm{mm}$ segments to be identified; only very minimal perceptual evidence of blackness was needed to choose the 2-mm segments. Ergo, this discrimination is a detection (rather than an identification) task. In conclusion, the very narrow rectangle's two vertical lines (context) increased the visibility of its component 2-mm segments; a context-produced increase in visibility occurred. Therefore, the Experiment 3 result that the very narrow rectangle was relatively unlikely to be falsely identified as having absent $2-\mathrm{mm}$ segments should have been due to the same context-produced increase in visibility.
Context-produced interference is more typical. Contextual objects interfere with the identification of target objects (Eriksen \& Hoffman, 1972; Kahneman, Treisman, \& Burkell, 1983; research on "crowding" reviewed in Strasburger, Harvey, \& Rentschler, 1991). In addition, targets are identified better when they are single objects than when they are components of a perceived (apprehended) unit (e.g., Prinzmetal \& Banks, 1977).

\section{GENERAL DISCUSSION}

\section{The Two Effects of Context}

One effect of context was that it reversed the closure that the salience-independence theory assumes is produced by a salient closure emergent feature. It was concluded that this closure was not due to this emergent feature, which is in opposition to the salience-independence theory.

The second effect of context was a context-produced increase in visibility. This effect was unexpected. Moreover, it seems to be new (below). Furthermore, it indicates that elemental features (two lines) affect the perception of other elemental features (two segments), even though it is not regularly assumed that perceptual primitives affect the perception of one another (e.g., Julesz, 1984; Treisman, 1986, pp. 29-32). Similarly, suppose that one line of a right angle increases the visibility of its second line (and/or vice versa). Then, it would not be clear in what sense a right angle is a perceptual primitive, even though it meets the illusory conjunction criterion for perceptual primitives (Treisman, 1986; Treisman \& Paterson, 1984).

So, two effects of context were obtained that question the salience-independence theory and the related concept of perceptual primitives. A countersuggestion is that these two effects of context were due to a process occurring after the registration of perceptual primitives. However, the fact that a detection task was employed (Experiment 4) does not favor this suggestion. Furthermore, other findings question the concept of perceptual primitives. For example, one criterion for perceptual primitives is that they join to produce illusory conjunctions. Nevertheless, changing the objects that were targets radically affected the frequency of illusory conjunctions, despite the occurrence of the same physical stimulus, suggesting that illusory conjunctions were affected by a strategic process $(\mathrm{Bu}-$ tler, Mewhort, \& Browse, 1991).

\section{The Context-Produced Increase in Visibility}

The context-produced increase in visibility is important, because a comparable type of perceptual improvement may not be known. "Good" objects were discriminated better than were "poor" objects, when both types of objects contained identical component lines-an objectsuperiority effect (Weisstein \& Harris, 1974). In addition, objects were identified more accurately when they occurred in normal scenes than when they occurred in atypical scenes (Biederman, 1972; Biederman, Mezzanotte, 
\& Rabinowitz, 1982). Nevertheless, the contexts of the poor objects and atypical scenes could have interfered with the perception of individual objects; the difference in performance may be due to context-produced interference, rather than to context-produced improvement. Likewise, performance improves when the two targets of a dividedattention task belong to the same single perceived unit (Duncan, 1984; Pollatsek \& Digman, 1977), but this improvement may stem from a reduction in divided-attentionproduced interference.

The introduction to this paper indicated that performance on an $A$ versus $B$ discrimination was worse than performance on an $A+$ context versus $B+$ context discrimination (Pomerantz et al., 1977). Because the A and B objects of the A versus B discrimination occurred alone, this result cannot be attributed to context-produced interference. The same result was obtained with other A, B, and context objects (Pomerantz et al., 1977; Williams \& Weisstein, 1978). Nevertheless, the A and B objects were very similar, being mirror images and/or identical except for a small difference in spatial location. In contrast, the stimuli that led to the poorer performance in the present research-two lines and a blank-differ categorically.

Moreover, discriminating two lines from a blank is a detection task, implying that the very narrow rectangle's two vertical lines (context) improved the detection (not identification) of its $2-\mathrm{mm}$ segments. It is known that context can improve detection (Doyle \& Leach, 1988; Purcell \& Stewart, 1986, 1988, 1991). For example, the detection of the left-versus-right location (and hence not identification) of an upright face is better than that of an inverted face. However, these results are similar to some of the above results in that they may also be due to interference among the features of one type of stimuli (e.g., inverted faces), rather than to an improvement in the perception of the features of the second type of stimuli (e.g., upright faces). In addition, these results seem to involve the visibility of complete stimuli, rather than the visibility of component features such as the present 2-mm segments. Also, they were obtained with a poststimulus mask. In conclusion, the present context-produced increase-invisibility evidence seems new.

Perhaps context-produced increases in visibility occur only when the context and target are contiguous, as in the present research. In accord with this possibility, a large rectangle failed to inhibit the perception of a small rectangle when these rectangles were contiguous (King, 1990b). Perhaps context-produced increases in visibility are limited in some more serious way. However, it may be that context-produced increases in visibility are comparable in status to the other three extant types of contextual modification of the perception of features: inhibition, contrast, and assimilation. If so, then these modifications would be described by a $2 \times 2$ classification, type of effect (either on visibility or similarity) $\times$ direction of effect (in either of two opposing directions), which should have theoretical import.

\section{The Closure and Division Outcomes}

The closure and division outcomes remain to be considered. The closure outcome might be attributed to the good continuation of the top and bottom $5-\mathrm{mm}$ colinear lines of the two brackets. But the two upright rectangles contain the same colinear lines as the two brackets. Furthermore, these rectangles also contain the vertical lines that increase the visibility of the connecting $2-\mathrm{mm}$ segments. Therefore, the good-continuation explanation incorrectly predicts that the two upright rectangles also should have produced closure.

Coding theory (Buffart, Leeuwenberg, \& Restle, 1981; Leeuwenberg, 1968) holds that objects with many repeating features and many repeating relations between features tend to be perceived. Therefore, the closure outcome may have occurred because the square-like rectangle contains more repeating features than do the two brackets (partly because the lengths of the four sides of a rectangle repeat). In addition, the division outcome might have occurred because the two upright rectangles contain more repeating features than does the internal-lines rectangle (partly because the two upright rectangles are identical and hence their elements repeat). Consequently, coding theory accounts for both the closure and division outcomes. Similarly, more information should be required to describe the two brackets than the square-like rectangle, whereas less information should be required to describe the two upright rectangles than the internal-lines rectangle (Hochberg \& McAlister, 1953).

\section{REFERENCES}

Banks, W. P., \& KrajiceK, D. (1991). Perception. Annual Review of Psychology, 42, 305-331.

Biederman, I. (1972). Perceiving real-world scenes. Science, 177, 77-80.

Biederman, I., Mezzanotte, R. J., \& Rabinowitz, J. D. (1982). Scene perception: Detecting and judging objects undergoing relational violations. Cognitive Psychology, 14, 143-177.

Buffart, H., Leeuwenberg, E., \& Restle, F. (1981). Coding theory of visual pattern completion. Journal of Experimental Psychology: Human Perception \& Performance, 7, 241-274.

Butler, B. E., Mewhort, D. J. K., \& Browse, R. A. (1991). When do letter features migrate? A boundary condition for feature-integration theory. Perception \& Psychophysics, 49, 91-99.

Doy LE, J. R., \& LEACH, C. (1988). Word superiority in signal detection: Barely a glimpse, yet reading nonetheless. Cognitive Psychology, 20, 283-318.

DUNCAN, J. (1984). Selective attention and the organization of visual information. Journal of Experimental Psychology: General, 113, 501-517.

Eriksen, C. W., \& Hoffman, J. E. (1972). Temporal and spatial characteristics of selective encoding from visual displays. Perception \& Psychophysics, 12, 201-204.

GANz, L. (1966). Mechanism of the figural after-effects. Psychological Review, 73, 128-150.

Gillam, B. (1975). New evidence for "closure" in perception. Perception \& Psychophysics, 17, 521-524.

HochBerg, J., \& MCAlister, E. (1953). A quantitative approach to figural "goodness." Joumal of Experimental Psychology, 46, 361-364.

JulEsz, B. (1984). Toward an axiomatic theory of preattentive vision. In G. Edelman, W. Einer, \& W. Cowan (Eds.), Dynamic aspects of neocortical function (pp. 585-612). New York: Wiley. 
Kahneman, D., Treisman, A., \& Burkell, J. (1983). The cost of visual filtering. Joumal of Experimental Psychology: Human Perception \& Performance, 9, 510-522.

KING, D. L. (1989, November). Brief discrete lines were perceived as continuous. Paper presented at the meeting of the Psychonomic Society, Chicago, IL.

KING, D. L. (1990a), Gestalts are more closely associated with performance on a discrimination task than are component stimuli. American Joumal of Psychology, 103, 37-52.

KING, D. L. (1990b). A large rectangle delays the perception of a separate small rectangle. Perception \& Psychophysics, 47, 369-378.

KING, D. L. (1992). Discriminations between one and two lines. Manuscript submitted for publication.

LEEUWENBERG, E. (1968). Structural information of visual patterns. The Hague: Mouton.

Pollatsek, A., Digman, L. (1977). Dependent spatial channels in visual processing. Cognitive Psychology, 9, 326-352.

Pomerantz, J. R., \& Pristach, E. A. (1989). Emergent features, attention, and perceptual glue in visual form perception. Journal of $E x$ perimental Psychology: Human Perception \& Performance, 15. 635-649.

Pomerantz, J. R., Sager, L. C., \& Stoever, R. J. (1977). Perception of wholes and their component parts: Some configural superiority effects. Journal of Experimental Psychology: Human Perception \& Performance, 3, 422-435.

Prinzmetal, W., \& Banks, W. P. (1977). Good continuation affects visual detection. Perception \& Psychophysics, 21, 389-395.

PuRcell, D. G., \& Stewart, A. L. (1986). The face-detection effect. Bulletin of the Psychonomic Sociery, 24, 118-120.

Purcell, D. G., \& Stewart, A. L. (1988). The face-detection effect: Configuration enhances detection. Perception \& Psychophysics, 43, 355-366.
Purcell, D. G., Stewart, A. L. (1991). The object-detection effect: Configuration enhances perception. Perception \& Psychophysics, 50, 215-224.

Strasburger, H., Harvey, L. O., JR., \& Rentschler, 1. (199I), Contrast thresholds for identification of numeric characters in direct and eccentric view. Perception \& Psychophysics, 49, 495-508.

Treisman, A. (1986). Properties, parts, and objects. In K. R. Boff, L. Kaufman, \& J. P. Thomas (Eds.), Handbook of perception and human performance: Vol. 2. Cognitive processes and performance (pp. 35-1-35-70). New York: Wiley.

Treisman, A., \& Gelade, G. (1980). A feature-integration theory of attention. Cognitive Psychology, 12, 97-136.

Treisman, A., \& Paterson, R. (1984). Emergent features, attention, and object perception. Journal of Experimental Psychology: Human Perception \& Performance, 10, 12-31.

Treisman, A., \& SCHMIDT, H. (1982). Illusory conjunctions in the perception of objects. Cognitive Psychology, 14, 107-141.

Weisstein, N. (1972). Metacontrast. In D. Jameson \& L. M. Hurvich (Eds.), Handbook of sensory physiology: Vol. 7/4. Visual psychophysics (pp. 233-272). New York: Springer-Verlag.

Weisstein, N., \& HarRIs, C. S. (1974). Visual detection of line segments: An object superiority effect. Science, 186, 752-755.

Williams, A., \& WEISSTEIN, N. (1978). Line segments are perceived better in a coherent context than alone: An object-line effect in visual perception. Memory \& Cognition, 6, 85-90.

(Manuscript received January 17, 1992; revision accepted for publication October 12, 1992.$)$ 